\title{
Prognostic Implications of Pulmonary Hypertension due to Constrictive Pericarditis
}

\begin{abstract}
Constrictive pericarditis (CP) is a pathological process leading to scarring, fibrosis and thickening of the pericardial layers with subseguent compression of the cardiac chambers, resulting in diastolic dysfunction. Pericardiectomy remains the only acceptable and curative surgical procedure in most CP patients. Pulmonary hypertension $(\mathrm{PH})$ is defined as a mean pulmonary arterial systolic pressure greater than $25 \mathrm{mmHG}$ at rest and $30 \mathrm{mmHg}$ during exercise. The prevalence of $\mathrm{PH}$ in CP has previously been reported to be rare; as a result the main aim of this paper is to extensively review the prevalence of $\mathrm{PH}$ in $\mathrm{CP}$ patients.
\end{abstract}

Keywords: Constrictive pericarditis; Mean pulomary arterial systolic pressure; Pericardiectomy

\author{
Review Article \\ Volume 9 Issue 5 - 2017 \\ Mamotabo R Matshela ${ }^{1,2,3 *}$ \\ ${ }^{1}$ University of KwaZulu-Natal, Durban, South Africa \\ ${ }^{2}$ Mediclinic Heart Hospital, Pretoria, South Africa \\ ${ }^{3}$ London School of Economics and Political Science, UK \\ *Corresponding author: Mamotabo R Matshela MB CHB, \\ PHD, University of KwaZulu-Natal, Durban, South Africa, \\ Mediclinic Heart Hospital, Pretoria, South Africa, London \\ School of Economics and Political Science, UK, \\ Email:mamotabomatsh@gmail.com
}

Received: June 11, 2017 | Published: September 08, 2017

Abbreviations: CP: Constrictive Pericarditis; LVEF: Left Ventricular Ejection Fraction; mPASP: Mean Pulmonary Arterial Systolic Pressure; PH: Pulmonary Hypertension; RV: Right Ventricle; TR: Tricuspid Valve

\section{Introduction}

Constrictive pericarditis (CP) is commonly a symmetrical pathological process from scarring, fibrosis and thickening involving the parietal and visceral pericardial layers; due to its compressive phenomenon on the cardiac chambers, CP results in diastolic dysfunction [1-9]. Although CP is regarded as a chronic process, some patients may develop mild pericardial constriction which may resolve spontaneously within few weeks or months following the initial insult. Constrictive pericarditis is thought to be a rare cause of pulmonary hypertension $(\mathrm{PH})$; as a result the main objective of this paper is to review $\mathrm{PH}$ due to $\mathrm{CP}$.

\section{Overview of constrictive pericarditis}

Etiology of CP: Tuberculous pericarditis remains an important cause of CP in the developing world, however in developed countries prior pericarditis, idiopathic, prior cardiac surgery and radiotherapy in particular prior mediastinal radiation exposure are the common causes of CP. Other causes should also be evaluated which include systemic inflammatory or connective tissue disease, uremia particularly in patients with chronic renal failure or end-stage renal diseases, malignancy etc.

Clinical characteristics of CP: During the early stages of $\mathrm{CP}$, patients may remain completely asymptomatic and clinical signs may be subtle. However, in patients with established or advanced CP, the typical presentation is similarly to that of right heart failure. Where patients may present with lower extremity edema, elevated jugular venous pressure which is classically characterized by a prominent y-descent and Kussmal sign.

The role of echocardiography in CP: Echocardiography is the most easily accessible, cheap, non-invasive and readily available in almost every center; and is useful for both diagnostic and could also assist to exclude possible differential diagnoses. Echocardiography has evolved extensively in the recent past, with the introduction of tissue Doppler and speckle tracking imaging modalities which are useful in guiding treatment and also have important prognostic implications in CP patients.

Differential diagnosis of CP: although restrictive cardiomyopathy has been widely reported as the main mimic for $\mathrm{CP}$, other possible diagnosis should be intertained during the initial evaluation of patients suspicious of constriction, and these include dilated cardiomyopathies, pericardial effusion and cardiac tumours.

Treatment modalities for CP: Although the primary cause of constriction should be evaluated and managed accordingly, surgery in the form of a complete pericardiectomy remains a definitive and curative management strategy in patient with chronic CP.

\section{Pulmonary hypertension related to constrictive pericarditis}

Demographics characteristics and risk factors of pulmonary hypertension in constriction: Data on the prevalence and risk factors of $\mathrm{PH}$ in $\mathrm{CP}$ are still rather limited. The factors presumed to be associated with the occurrence or severity of $\mathrm{PH}$ in patients with $\mathrm{CP}$ include: old age, prior cardiac surgery, pre-existing $\mathrm{PH}$, severity and chronicity of $\mathrm{PH}$ at the time of surgery, primary etiology of CP particularly prior mediastinal radiotherapy, collagen vascular or connective tissue diseases, chronic respiratory diseases and pre-existing myocardial diseases [2,3,10]. Talreia et al. [11,12] reported comparison of etiologies between groups with $\mathrm{PH}$ and showed that prior cardiovascular surgery $(18 \%$ vs $38 \%)$ and irradiation (13\% vs $19 \%$ ) were more common in the $\mathrm{PH}$ group with mean pulmonary arterial systolic pressure (mPASP) of 
greater than $50 \mathrm{mmHg}$, while idiopathic (likely viral) etiology was more common in the PH group with mPASP of less than 50 $\mathrm{mmHg}$. Although left ventricular (LV) systolic dysfunction and the presence of valvular lesion are presumed to be among the leading causes or risk factors for PH in CP; Talreia et al. [11,12] reported no difference in $\mathrm{LV}$ ejection fraction ( $56 \%$ vs $58 \%$ ) or frequency of significant mitral incompetence ( $2 \%$ vs $0 \%$ ) or TR ( $5 \%$ vs $4 \%$ ), in patients with mPASP $>50 \mathrm{mmHg}$ versus $<50 \mathrm{mmHg}$. However, more data is warranted to address these controversies.

Mechanisms of pulmonary hypertension in constrictive pericarditis: In the true sense mPASP and right ventricular systolic pressures are estimated to be normally between 35 and $45 \mathrm{mmHg}$; however mPASP may be as low as possible reflecting reduced RV stroke or pressure and sometimes mildly elevated if the left atrial pressure has caused a secondary increase in pulmonary vascular resistance. In rare instances localized constriction can cause external valvular constriction or pinching of the epicardial coronaries. Up until recently, there has been case reports in patients with severe $\mathrm{PH}$ in the setting of $\mathrm{CP}$ [10]. Severe $\mathrm{PH}$ has been reported as a rare feature of $\mathrm{CP}$ and may indicate coexisting cardiac or pulmonary disease, particularly in patient with prior cardiac surgery or radiotherapy.

Talreia et al. [13] reported a case of severe $\mathrm{PH}$ due to $\mathrm{CP}$ in a $58 y e a r s$ old female, who had a reversible constriction associated with chronic pleural effusion and hypoxemia [10]. The magnitude of mPASP improved significantly before and after pericardiectomy following the initiation of medical treatment with sildenafil and nitric oxide. This approach remains controversial as there is a paucity of evidence regarding the optimal medical management of PH secondary to CP. Treatment of $\mathrm{PH}$ in $\mathrm{CP}$ patients using pulmonary vasodilators may be hazardous given their potential to increase LV filling pressures further, thus worsening pulmonary edema. Brunner et al. $[10,14]$ reported a case where there was deterioration in a patient's condition after the initiation of sildenafil.

Impact of percicardiectomy on pulmonary hypertension in constriction: Pericardiectomy remains the only curative treatment for CP, irrespective of the chronicity or severity of constriction. Reports have highlighted the impact of pericardiectomy on $\mathrm{PH}$ in patients with surgery or autopsy confirmed CP [3]. In the same reports pericardiectomy significantly improves the level of $\mathrm{PH}$ in those who survived surgery [3]. However, long-term follow-up data on patients undergoing surgery with pre-operative severe $\mathrm{PH}$ are still limited, as a result this opens a window of opportunities to further study these patients in long-term.

Prognosis in constriction patient with pulmonary hypertension: Long-term survival after pericardiectomy for $\mathrm{CP}$ is related to the underlying etiology, left ventricular systolic function, renal function, serum sodium and pulmonary pressures. High pulmonary pressures may reflect the severity of constriction, concomitant myocardial dysfunction or pulmonary pathology. Talreia et al. [11] reported data of 224 patients with surgically or autopsy confirmed CP, where $20 \%$ of these patients had PH. Pericardioectomy significantly improved the level of mPASP and survival in those who survived the surgical procedure. Improvement on survival for those with $\mathrm{PH}$ was almost similar to their counterparts with normal preoperative mPASP. Furthermore, Talreia et al. [11] demonstrated a significantly higher perioperative or 30 day mortality particularly in the group with higher mPASP, mostly the group with mPASP of more than 50 $\mathrm{mmHg}$. This group consisted mainly of patients with prior cardiac surgery or mediastinal radiotherapy.

\section{Future directions}

Each patient with $\mathrm{PH}$ presumed to be due to $\mathrm{CP}$ should be properly evaluated and managed individually as the presence of PH in CP may declare poor prognostic in some patients. Data are still limited on mechanisms and outcome; as a result a window of opportunities is opened for future research to address many unanswered questions related to the pathological mechanisms and long-term implications of $\mathrm{PH}$ in $\mathrm{CP}$ irrespective of the primary etiology of $\mathrm{CP}$.

\section{Conclusion}

Although the prevalence $\mathrm{PH}$ in $\mathrm{CP}$ is presumed to be rare, $\mathrm{PH}$ due to $\mathrm{CP}$ could be a reversible entity with use of vasodilatation and pericardiectomy. Pericardiectomy seems to improve the level mPASP and survival in those who survive surgery, similarly to those with normal baseline mPASP; however larger studies with longer follow-up are still warranted.

\section{Acknowledgement}

None.

\section{Funding}

None.

\section{Disclosures}

None related to this study.

\section{References}

1. Miranda WR, Oh JK (2017) Constrictive Pericarditis: A Practical Clinical Approach. Prog Cardiovasc Dis 59(4): 369-379.

2. Ling LH, Oh JK, Schaff HV, Danielson GK, Mahoney DW, et al. (1999) Constrictive pericarditis in the modern era - Evolving clinical spectrum and impact on outcome after pericardiectomy. Circulation 100(13): 1380-1386.

3. Ling LH, Oh JK, Danielson GK, Schaff HV, Mahoney DW, et al. (1997) Outcome following pericardiectomy for constrictive pericarditis: Influence of an evolving disease. Circulation 96: 158-158.

4. Veinot JP, Edwards WD (1996) Pathology of radiation-induced heart disease: A surgical and autopsy study of 27 cases. Hum Pathol 27(8): 766-773.

5. Matshela MR, Blauwet LA, Pellikka PA, Espinosa RE, Greason KL, et al. (2014) Pericardiectomy improves right ventricular echocardiographic mechanics in patients with constrictive pericarditis without history of radiotherapy or previous cardiac surgery. Eur Heart J 35: 207-207.

6. Tritto M, Calabrese P (1996) Radiation-induced heart disease. Oncol Rep 3: 21-26. 
7. Ak K, Demirbas E, Atas H, Birkan Y, Akalin F, et al. (2016) Results of pericardiectomy for constrictive pericarditis Single-center experience. Herz 42(1): 75-83.

8. Depboylu BC, Mootoosamy P, Vistarini N, Testuz A, El-Hamamsy I, et al. (2017) Surgical Treatment of Constrictive Pericarditis. Tex Heart I J 44(2): 101-106.

9. Lin YY, Zhou M, Xiao J, Wang B, Wang ZN (2012) Treating Constrictive Pericarditis in a Chinese Single-Center Study: A Five-Year Experience. Ann Thorac Surg 94(4): 1235-1240.

10. Brunner NW, Ramachandran K, Kudelko KT, Sung YK, Spiekerkoetter E, et al. (2013) A case of recurrent pericardial constriction presenting with severe pulmonary hypertension. Pulm Circ 3(2): 436-439.

11. Talreia DR, Nishimura RA, Oh J, Appleton C, Higano ST, et al. (2002) Constrictive pericarditis in the modern era: Novel criteria for diagnosis in the cardiac catheterization laboratory. J Am Coll Cardiol 39: 149a-149a.
12. Talreia DR, Edwards WD, Oh J, Tazelaar H, Danielson G (2002) Constrictive pericarditis with normal pericardial thickness on examination of histopathology. J Am Coll Cardiol 39: 157a-157a.

13. Tajena A, James K, Timothy W, Alfred N, Kenneth P (2010) Unusual case of chronic constrictive pericarditis complicated by severe pulmonary arterial hypertension. Am J Respir Crit Care Med 181: A3409.

14. Brunner L, Hoffmeister HE, Koncz J, Stapenhorst K (1966) [False diagnosis in constrictive pericarditis. Survey of clinical findings in 27 cases]. Med Klin 61: 1283-1286. 